\title{
Right Ventricular Free wall Longitudinal Strain in Heart Failure with Mid-range and Preserved Ejection Fraction. Application and Prognostic Implications.
}

Daniel Grados-Saso ( $\sim$ danielgrados_87@hotmail.com )

Hospital de Barbastro

Juan Manuel Salvador

Hospital de Barbastro

Anyuli Gracia-Gutiérrez

Centro Universitario de la Defensa Zaragoza

Jorge Rubio-Gracia

Lozano Blesa University Clinical Hospital: Hospital Clinico Universitario Lozano Blesa

Juan Ignacio Perez-Calvo

Hospital Clínico Universitario Lozano Blesa: Hospital Clinico Universitario Lozano Blesa

Isaac Lacambra-Blasco

Lozano Blesa University Clinical Hospital: Hospital Clinico Universitario Lozano Blesa

\section{Research Article}

Keywords: Heart failure, Right ventricular free wall longitudinal strain, Heart failure with mid-range ejection fraction

Posted Date: February 10th, 2021

DOI: https://doi.org/10.21203/rs.3.rs-180622/v1

License: (c) (i) This work is licensed under a Creative Commons Attribution 4.0 International License.

Read Full License 


\title{
RIGHT VENTRICULAR FREE WALL LONGITUDINAL STRAIN IN HEART
}

\section{FAILURE WITH MID-RANGE AND PRESERVED EJECTION FRACTION.}

\section{APPLICATIONS AND PROGNOSTIC IMPLICATIONS.}

\author{
ABBREVIATIONS \\ FAC Fractional area change \\ LVEF Left ventricular ejection fraction \\ HF Heart failure \\ HFmrEF Heart failure with mid-range ejection fraction \\ HFpEF Heart failure with preserved ejection fraction \\ VTI Velocity time integral \\ PASP Pulmonary artery systolic pressure \\ LVGLS Left ventricular global longitudinal strain \\ RVFWLS Right ventricular free wall longitudinal strain \\ TAPSE Tricuspid annular plane systolic excursion \\ ICT Isovolumetric contraction time \\ IVRT Isovolumetric relaxation time \\ ET Ejection time \\ RVOT Right ventricle outflow tract \\ RV Right ventricle
}

\section{INTRODUCTION:}

Right ventricle plays an important role in the pathophysiology of heart failure with preserved and midrange ejection fraction. Right ventricular dysfunction is associated with an increase in morbidity and mortality in this population $(1,2)$. The gold standard method for volume and functional evaluation of the right ventricle is magnetic resonance imaging (MRI) (3). However, the transthoracic echocardiogram is more commonly used in clinical practice due to its greater accessibility. Quantification of right ventricular functional parameters by echocardiography is challenging due to the complex geometry of this heart chamber (4). Several methods are available to evaluate its function: Tricuspid annular plane systolic excursion (TAPSE), ventricular fractional area change (FAC), lateral tricuspid annular systolic velocity derived from tissue-Doppler (S ') and Tei index (5). These parameters, largely used for decades and of proven utility, present an important limitation: their values are dependent on the insonation angle and can be affected by changes in load as well as rotational and translational motions of the heart, especially present in the right ventricle (6).

Echocardiography provides an additional tool, such as the determination of RV strain, using techniques to study RV deformation that could be more sensitive to detect right ventricular dysfunction. Speckletracking programs are used to measure the three segments of the RV free wall. The lower limit of normality has been set at $<-20 \%$ (greater than 20 in absolute value) $(7,8)$. In patients with heart failure with preserved ejection fraction, an altered right ventricular function is common (9) and similarly to the left ventricle, there is most likely an association with the same fibrosis processes that affect the subendocardial layer in relation to the various prevalent comorbidities in HFpEF and HFmrEF. In 
addition, right ventricular dysfunction could contribute to the symptomatology of heart failure, with worse symptomatic control, more admissions and worse clinical outcome (10)

We aim to evaluate right ventricular function by determining right ventricular free wall strain in addition to TAPSE, S' wave, Tei index and FAC in a cohort of patients admitted for an episode of heart failure with preserved and mid-range ejection fraction (HFpEF and HFmrEF), evaluating their relation with variables such as hospital readmission and cardiovascular mortality.

\section{MATERIALS AND METHODS}

A prospective cohort study was designed made up of patients admitted to hospital for clinical heart failure. A complete doppler echocardiogram was performed according to the usual practice of our echocardiography laboratory. Measurements of the various parameters were performed following the recommendations of the quantification consensus guide of the European and American Echocardiography Societies. All studies were performed with the Phillips Epiq 7C cardiovascular ultrasound machine using a matrix probe of 1-4 MHz. Among other parameters, the following were obtained from the echocardiogram: RV dimensions and function data: TAPSE in M-mode of the lateral tricuspid annulus, tissue Doppler S' wave of the lateral tricuspid annulus, fractional area change, Tei index and RV free wall strain, LV longitudinal strain, left ventricular ejection fraction using the bi-plane method of disc summation (Simpsons method). Other parameters were also obtained such as PASP that was estimated by continuous-wave Doppler registration of the maximum flow velocity of tricuspid regurgitation when the signal quality was adequate for a reliable estimate. Tei index was calculated through tissue Doppler signal of the lateral tricuspid annulus, by adding the isovolumic contraction time and isovolumic relaxation time and dividing them by the right ventricular ejection time. The fractional area change was calculated using two-dimensional imaging with a plane focused on the right ventricle, outlining the area in end-diastole and end-systole. For deformation analysis, the aCMQ tool from Phillips ${ }^{\mathrm{R}}$ was used for the semi-automatic calculation of strain. For the analysis of the LV longitudinal strain from the apical three-, four- and twochamber views, automatic detection of endocardial borders and aortic valve closure time were carried out. End-diastole was identified either through the QRS complex or as the first frame after mitral valve closure. The software tracked the acoustic signals throughout the cardiac cycle. Left ventricular global longitudinal strain was automatically calculated by generating regional data from 6 segments and a mean value for each view.Adequate monitoring of myocardial segments was checked by the researcher. In the event that a segment was not properly followed, it was corrected and if suboptimal monitoring of the endocardial border persisted throughout the cardiac cycle in a segment, it was eliminated for analysis of the overall computation. At most, the elimination of two segments from the LV 17-segment model was accepted. In the event of having to delete more than two segments, the result of the strain was considered not valid for the analysis and was therefore excluded.

To determine right ventricular free wall strain (RVFWS), a 4-chamber view centered on right chambers was obtained and the image was optimized to obtain the maximum resolution with the greatest visualization of the entire RV free wall. The analysis tool available for the LV was used, as there is no specific tool for RV strain. Through the 6-segment model of the LV in 4-chamber apical view, three segments of the RV free wall were analyzed for the calculation of peak strain mean value for all three free wall segments (basal, middle and apical) and free wall RVLS (Figure 1). Interventricular septum segments were excluded from the calculation. In case of suboptimal monitoring of any segment throughout the cardiac cycle, it was corrected for better follow-up. Exclusion of any of the three RV free wall segments was not accepted. All studies were stored digitally. 


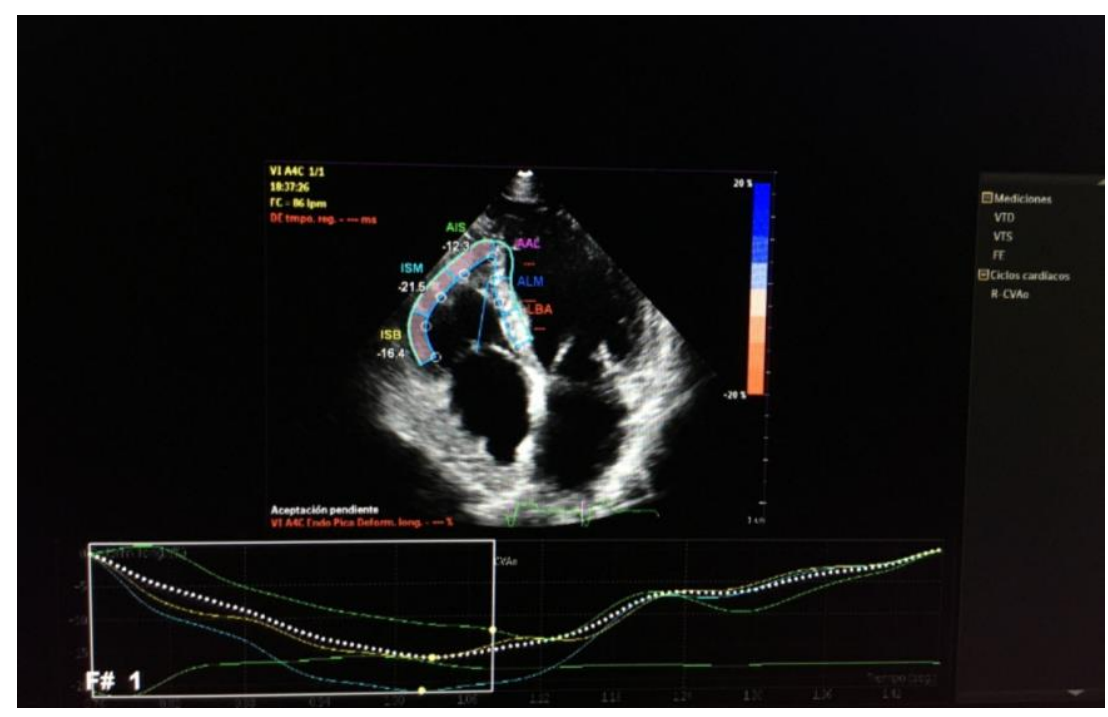

Figure 1. Determination of the right ventricular free wall strain through two-dimensional speckle tracking echocardiogram

Some parameters may be altered during the acute phase of heart failure by hemodynamic changes activated as a compensatory mechanism in the most initial phase of decompensated HF. Therefore, the echocardiogram of the index admission was performed prior to hospital discharge once the heart failure situation had stabilized. That situation defined as: clinical improvement of congestive semiology (a subjective improvement with decreased dyspnea, edema, pulmonary auscultation) at least $24 \mathrm{~h}$ after the patient's admission. Inclusion criteria were the following: patients admitted to hospital with an episode of heart failure, with preserved and mid-range ejection fraction (measured by the bi-plane Simpson method at $>40 \%$ ). Exclusion criteria were: significant heart valve disease (mitral, tricuspid or aortic regurgitation equal to or greater than grade III/IV), severe mitral or aortic stenosis; intervened valvular heart disease:prosthetic heart valves or mitral or tricuspid prosthetic valve rings; cardiomyopathy, autoimmune disease, connective tissue disease or diagnosed active neoplasm; low LV ejection fraction (LVEF $<40 \%)$; refusal to enter the study or no signed informed consent; and lastly poor acoustic window that prevented reliable calculation of the strain.

The study was approved by Hospital ethical committee of clinical research. Patients were followed for six months. Readmissions for HF and deaths (from cardiovascular and non-cardiovascular causes) were recorded.

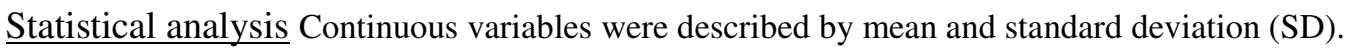
Qualitative or categorical variables are presented by frequency distribution of the percentages in each category. Quantitative variables were explored with the Shapiro-Wilk normality test (measures of goodness-of-fit measure to a normal distribution) and indicators of central tendency (mean or median) and dispersion (standard deviation or percentiles) are given.

The association between factors was assessed by statistical hypothesis testing using proportion comparison when both variables were qualitative (chi square test, Fisher's exact test), mean comparison when one them was quantitative (Student t-test, ANOVA test, and if they didn't follow normal distribution then mann-Whitney U test or Kruskal-Wallis test) and bivariate correlation (Pearson correlation coeficient) when both were quantitative or, if the conditions of application were not met, Spearman's correlation. In order to evaluate the contribution of RV free wall strain to mortality while controlling possible confounding factors, multivariate logistic regression models were performed. The effects were considered significant if $\mathrm{p}<0.05$. The two-tailed $\mathrm{p}$ values and the power of all statistical tests were taken into account when drawing conclusions. 


\section{RESULTS}

Between April 2018 and July 2019 patients were consecutively included in the study. The total study population was initially 143 patients, of which 5 were excluded for presenting significant valvular disease ( 3 with moderate to severe or severe mitral regurgitation (from grade III/IV) and 2 with severe aortic stenosis), 15 were excluded with a poor echocardiographic window, unable to follow up or refusal to sign informed consent, leaving 125 patients, of whom 54 were excluded for presenting $\mathrm{LVEF}<40 \%$. Thus, 71 patients were included in the analysis. The data were collected in a database with the SPSS version 15.0 program and analyzed with $\mathrm{R}$ statistical analysis.

\section{$\underline{\text { Clinical characteristics }}$}

Baseline clinical characteristics are summarized in Table 1

\begin{tabular}{|l|c|}
\hline \multicolumn{1}{|c|}{ Clinical history } & Percentage (number of patients) \\
\hline Men & $55 \%$ \\
\hline Women & $45 \%$ \\
\hline Mean age years (SD) & $77,58(10,75)$ \\
\hline Kidney failure & $38,03 \%(27)$ \\
\hline Dyslipidemia & $50 \%(35)$ \\
\hline Type 2 diabetes & $42.25 \%(30)$ \\
\hline Smoking & $7.14 \%(5)$ \\
\hline COPD & $19.72 \%(14)$ \\
\hline Atrial fibrillation & $54.93 \%(39)$ \\
\hline HBP & $92,96 \%(66)$ \\
\hline Ischemic heart disease & $35 \%(25)$ \\
\hline
\end{tabular}

Table $1 . \%$ (number of patients)

The average body mass index (BMI) was 30.23 (4.8 SD) in women and 29.49 (5.38 SD) in men.

The medical treatment received by patients is shown in Table 2 .

\begin{tabular}{|c|c|}
\hline Medical treatment & Percentage \\
\hline ACE inhibitor & $29 \%$ \\
\hline ARBs & $32 \%$ \\
\hline MCRA & $19 \%$ \\
\hline Beta blocker & $60 \%$ \\
\hline Furosemide & $62 \%$ \\
\hline Digoxin & $19 \%$ \\
\hline Thiazide & $15 \%$ \\
\hline
\end{tabular}

Table 2. Medical treatment

Echocardiographic variables: The main echocardiographic variables are listed in Table 3. 


\begin{tabular}{|c|c|}
\hline Parameter & \\
\hline $\begin{array}{l}\text { Proximal RVOT diameter } \\
(\mathrm{mm})\end{array}$ & 29,24 (SD 5.47) \\
\hline $\begin{array}{l}\text { Distal RVOT diameter } \\
(\mathrm{mm})\end{array}$ & 23.34 (SD 4.24) \\
\hline $\begin{array}{l}\text { Basal RV diameter apical } \\
4 \mathrm{c}(\mathrm{mm})\end{array}$ & 39,66 (SD 5.51) \\
\hline $\begin{array}{l}\text { Mean RV diameter apical } \\
4 \mathrm{c}(\mathrm{mm})\end{array}$ & 30.12 (SD 6.27) \\
\hline Right atrium area $\left(\mathrm{cm}^{2}\right)$ & 21.89 (SD 6.10) \\
\hline TAPSE (mm) & $19.03(\mathrm{SD} 3.36)$ \\
\hline FAC (\%) & $52,6 \%$ \\
\hline Tei Index & 0.51 (SD 0.13) \\
\hline RVFWLS (\%) & $17.29 \%(\mathrm{SD} 4.41)$ \\
\hline $\begin{array}{l}\text { Pulmonary velocity } \\
\text { acceleration time (msec) }\end{array}$ & 87,09 (SD 19.56) \\
\hline Pulmonary flow VTI $(\mathrm{cm})$ & 15.20 (SD 4.39) \\
\hline PASP $(\mathrm{mmHg})$ & $48.20($ SD 16.48) \\
\hline LVEF & $55,1 \%(\mathrm{SD} 8,64)$ \\
\hline LVGLS\% & $14.27(3.39)$ \\
\hline $\begin{array}{l}\operatorname{mrLVEF}(40-50 \%) / \\
\operatorname{pLVEF}(>50 \%)\end{array}$ & $35 / 65$ \\
\hline
\end{tabular}

Table 3

Echocardiographic variables. Distal RVOT (Distal right ventricular outflow tract), FAC (Fractional area change), LVGLS (left ventricular global longitudinal strain, absolute value), $R V F W L S$ (right ventricular free-wall longitudinal strain). mrLVEF (mid-range LVEF)/ $\mathrm{pLVEF}$ (preserved LVEF) \% (percentage)

Most of the patients showed RV longitudinal dysfunction revealed by longitudinal strain: the percentage of patients with decreased RV strain (less than $20 \%$ in absolute value) was $63 \%$. In the vast majority of patients LV longitudinal function evaluated by global longitudinal strain was also impaired: the percentage of patients with decreased GLS (less than $18 \%$ in absolute value) was $77 \%$.

\section{Events}

Within 6 months of inclusion in the study, 33.80\% of patients had been readmitted to hospital with heart failure and $14 \%$ of patients had died of cardiovascular causes (heart failure or myocardial infarction, sudden death). $4,2 \%$ of patients died of non-cardiovascular causes in the first 6 months. Among the causes of death, $77 \%$ died from cardiovascular causes $(72 \%$ of patients from heart failure, $5 \%$ from sudden death), $5 \%$ from kidney failure and $18 \%$ from neoplasia. Average time until readmission with heart failure was 56,19 days while the average time until death from HF was 114,35 days. 
The presence of kidney failure (OR 4.7 [1.66;14.2] p 0.003), with a higher concentration of creatinine (OR 2.95 [1.29;6.6.75] p 0.011) and a lower GF were associated with increased likelihood of hospital admission with heart failure, while renal failure and lower glomerular filtration were associated to cardiovascular mortality at 6 months (Table 4 and 5). Hypertension, type 1 diabetes, type 2 diabetes, smoking, COPD, dyslipemia, history of ischemic heart disease (revascularized and non-revascularized) and atrial fibrillation were not associated to a higher likelihood of readmission with HF or higher mortality within the first 6 months of inclusion.

\begin{tabular}{|l|c|c|c|}
\hline Clinical history & Not readmission & Readmission & $p$ \\
\hline Renal failure & $12(26 \%)$ & $15(63 \%)$ & 0,03 \\
\hline Creatinine & $1.05[0.86 ; 1.29]$ & $1.60[1.02 ; 1.84]$ & 0,011 \\
\hline GF & $66.00[59.17 ; 78.64]$ & $40.53[30.58 ; 50.55]$ & $<0,001$ \\
\hline Hypertension & $44(94 \%)$ & $22(92 \%)$ & 0,7 \\
\hline Diabetes mellitus & $22(47 \%)$ & $8(33 \%)$ & 0,29 \\
\hline Smoking & $3(6 \%)$ & $2(9 \%)$ & 0,730 \\
\hline COPD & $8(17 \%)$ & $6(25 \%)$ & 0,44 \\
\hline Dyslipemia & $21(47 \%)$ & $14(58 \%)$ & 0,329 \\
\hline Ischemic heart disease & $9(19 \%)$ & $3(12 \%)$ & 0,322 \\
\hline Atrial fibrillation & $28(60 \%)$ & $11(46 \%)$ & 0,286 \\
\hline
\end{tabular}

Table 4 Clinical history- Readmission with heart failure at 6 months., GF (glomerular filtration), HBP (high blood pressure),

\begin{tabular}{|l|c|c|c|}
\hline Cliical history & Not death & Death & $p$ \\
\hline Renal failure & $20(34 \%)$ & $7(70 \%)$ & 0,035 \\
\hline Creatinine & $1.07[0.89 ; 1.38]$ & $1.65[1.18 ; 1.76]$ & 0,15 \\
\hline GF & $63.12[42.50 ; 76.08]$ & $35.80[31.00 ; 45.00]$ & $<0,022$ \\
\hline Hypertension & $57(93 \%)$ & $9(90 \%)$ & 0,6 \\
\hline Diabetes mellitus & $26(43 \%)$ & $4(40 \%)$ & 0,89 \\
\hline COPD & $11(18 \%)$ & $3(30 \%)$ & 0,48 \\
\hline Dyslipemia & $29(48 \%)$ & $6(60 \%)$ & 0,52 \\
\hline Ischemic heart disease & $9(15 \%)$ & $3(30 \%)$ & 0,526 \\
\hline Atrial fibrillation & $33(54 \%)$ & $6(60 \%)$ & 0,749 \\
\hline
\end{tabular}

Table 5 Cardiovascular mortality at 6 months., GF (glomerular filtration)

Neither TAPSE nor FAC nor S wave were associated with a higher probability of readmission or mortality. A lower Tei index was associated to lower chance of readmission and mortality, while in patients with adverse events a wide dispersion of Tei index was found (0.5 to 0.75$)$.

A worse right ventricular free wall longitudinal strain (free wall RVLS) was related to higher cardiovascular mortality at 6 months (18,9 vs 13,96 (OR $0.86[0.74 ; 1.00$, p 0.049) (table 7).

As to left ventricular functional parameters, septal mitral E/e' ratio, average E/e' ratio (septal and lateral), and LVGLS also showed higher cardiovascular mortality at 6 months. Ejection fraction showed no relation to readmission or mortality (table 7).

Admission with HF at 6 months

\begin{tabular}{|c|c|c|c|c|c|}
\hline & \{total $]$ & no & yes & OR & p.ratio \\
\hline & $N=71$ & $N=47$ & $N=24$ & & \\
\hline RVFWLS & $18.70[13.73 ; 20.73]$ & $18.83[14.08 ; 20.84]$ & $18.40[12.85 ; 20.65]$ & $0.96[0.86 ; 1.08]$ & 0.516 \\
\hline maxVTI & $3.02[2.70 ; 3.30]$ & $3.05[2.70 ; 3.30]$ & $3.00[2.75 ; 3.28]$ & $1.11[0.40 ; 3.03]$ & 0.843 \\
\hline TAPSE & $18.05[17.07 ; 21.00]$ & $18.00[17.35 ; 21.00]$ & $18.40[15.82 ; 20.65]$ & $0.97[0.84 ; 1.13]$ & 0.733 \\
\hline S & $11.00[10.00 ; 12.00]$ & $11.00[9.50 ; 12.00]$ & $11.00[10.00 ; 11.00]$ & $1.07[0.85 ; 1.35]$ & 0.566 \\
\hline FAC & $0.52[0.46 ; 0.62]$ & $0.52[0.45 ; 0.60]$ & $0.55[0.50 ; 0.66]$ & $0.98[0.94 ; 1.01]$ & 0.212 \\
\hline
\end{tabular}


Admission with $\mathrm{HF}$ at 6 months

\begin{tabular}{|c|c|c|c|c|c|}
\hline & \{total $]$ & no & yes & OR & p.ratio \\
\hline TeiRV & $0.47[0.43 ; 0.56]$ & $0.46[0.41 ; 0.51]$ & $0.56[0.45 ; 0.73]$ & $1457[6.75 ; 314540]$ & 0.008 \\
\hline E/e' & $15.95[12.70 ; 22.08]$ & $15.10[11.80 ; 21.00]$ & $19.60[15.40 ; 23.00]$ & $1.09[1.01 ; 1.17]$ & 0.027 \\
\hline LVEF & $56.86[47.86 ; 61.14]$ & $56.38[48.12 ; 60.56]$ & $57.42[47.86 ; 61.96]$ & $1.01[0.95 ; 1.07]$ & 0.835 \\
\hline LVGLS & $14.50[12.00 ; 16.60]$ & $14.50[12.20 ; 16.85]$ & $14.00[10.60 ; 15.93]$ & $0.89[0.77 ; 1.04]$ & 0.140 \\
\hline PASP & $47.00[37.00 ; 53.00]$ & $47.00[34.25 ; 51.50]$ & $46.00[39.50 ; 54.50]$ & $1.01[0.98 ; 1.04]$ & 0.65 \\
\hline \hline E/e ${ }_{\text {average }}$ & $15.10[12.73 ; 18.30]$ & $13.82[10.98 ; 18.06]$ & $15.60[13.85 ; 19.00]$ & $1.12[1.00 ; 1.24]$ & 0.043 \\
\hline
\end{tabular}

Cardiovascular mortality at 6 months

\begin{tabular}{|c|c|c|c|c|c|}
\hline & [total] & no & yes & OR & p.ratio \\
\hline & $N=71$ & $N=61$ & $N=10$ & & \\
\hline E/e' & $15.95[12.70 ; 22.08]$ & $15.40[12.50 ; 20.60]$ & $27.00[18.60 ; 31.60]$ & $1.11[1.02 ; 1.21]$ & 0.015 \\
\hline E/e ${ }_{\text {average }}$ & $15.10[12.73 ; 18.30]$ & $13.85[11.78 ; 17.25]$ & $21.85[18.24 ; 28.61]$ & $1.15[1.02 ; 1.29]$ & 0.025 \\
\hline LVGLS & $14.50[12.00 ; 16.60]$ & $15.10[12.20 ; 16.70]$ & $11.40[10.60 ; 14.50]$ & $0.76[0.60 ; 0.96]$ & 0.021 \\
\hline RVFWLS & $18.70[13.73 ; 20.73]$ & $18.90[14.00 ; 20.90]$ & $13.96[10.83 ; 18.82]$ & $0.86[0.74 ; 1.00]$ & 0.049 \\
\hline maxVTI & $3.02[2.70 ; 3.30]$ & $3.00[2.70 ; 3.30]$ & $3.16[3.04 ; 3.45]$ & $3.29[0.93 ; 11.6]$ & 0.064 \\
\hline TAPSE & $18.05[17.07 ; 21.00]$ & $18.05[17.23 ; 21.00]$ & $18.25[16.90 ; 22.00]$ & $1.00[0.81 ; 1.22]$ & 0.972 \\
\hline S & $11.00[10.00 ; 12.00]$ & $11.00[10.00 ; 12.00]$ & $10.00[9.00 ; 11.00]$ & $0.84[0.58 ; 1.20]$ & 0.337 \\
\hline FAC & $0.52[0.46 ; 0.62]$ & $0.52[0.45 ; 0.61]$ & $0.67[0.51 ; 0.73]$ & $1.00[0.97 ; 1.04]$ & 0.867 \\
\hline TeiRV & $0.47[0.43 ; 0.56]$ & $0.47[0.42 ; 0.56]$ & $0.73[0.60 ; 0.74]$ & $1456[2.46 ; 863430]$ & 0.025 \\
\hline PASP & $47.00[37.00 ; 53.00]$ & $45.50[34.75 ; 50.50]$ & $50.00[42.00 ; 55.00]$ & $1.03[0.99 ; 1.08]$ & 0.088 \\
\hline
\end{tabular}

Table 7 Echocardiogram parameters. Cardiovascular mortality at 6 months. TAPSE (tricuspid annular plane systolic excursion), FAC (fractional area change), S (tricuspid TDI S wave), RVFWLS (right ventricular strain), Ele' (mitral E wave and mitral e' tissue wave). $E / e^{\prime}$ average (Septal and lateral E/e' average).

In the linear regression analysis, significant $(\mathrm{p}<0.01)$ linear correlation (Pearson r 0.45, CI 0.246-0.619) was observed between the longitudinal deformation indices of both ventricles (Figure 2)

\section{SLGVI}

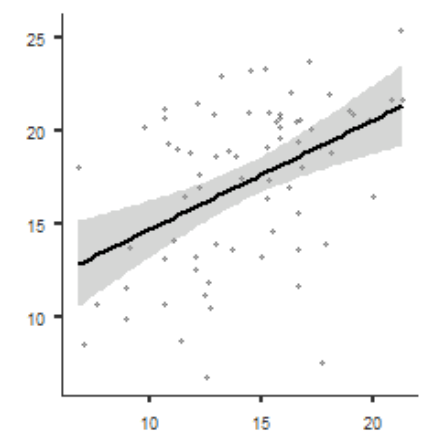


In a multivariate analysis that included age, BMI, e/E ratio, ejection fraction (LVEF) and AF (established risk factors of mortality in heart failure with preserved LVEF) the altered RV free wall strain remained a predictor of cardiovascular mortality at 6 months.

Cardiovascular death at 6 months

\begin{tabular}{|c|l|}
\hline CVdeath6m & \\
\hline RVFWLS & Mean (SD) \\
\hline Age & Mean (SD) \\
\hline BMI & Mean (SD) \\
\hline E/e' & Mean (SD) \\
\hline E/e' average & Mean (SD) \\
\hline LVEF & Mean (SD) \\
\hline AF & no \\
\hline & yes \\
\hline
\end{tabular}

\begin{tabular}{rr|c}
\multicolumn{1}{c|}{ no } & \multicolumn{1}{c|}{ yes } & OR (univariate) \\
$17.7(4.3)$ & $14.7(4.6)$ & $0.86(0.73-1.00, \mathrm{p}=0.049)$ \\
$77.0(10.9)$ & $81.2(9.3)$ & $1.04(0.98-1.13, \mathrm{p}=0.250)$ \\
$29.5(4.8)$ & $32.5(6.7)$ & $1.11(0.98-1.26, \mathrm{p}=0.101)$ \\
$17.1(7.3)$ & $25.1(9.1)$ & $1.11(1.03-1.22, \mathrm{p}=0.015)$ \\
$15.0(6.0)$ & $26.2(13.7)$ & $1.15(1.04-1.34, \mathrm{p}=0.025)$ \\
$54.3(8.7)$ & $59.5(7.0)$ & $1.08(0.99-1.18, \mathrm{p}=0.090)$ \\
$28(87.5)$ & $4(12.5)$ & - \\
$33(84.6)$ & $6(15.4)$ & $1.27(0.33-5.40, \mathrm{p}=0.729)$
\end{tabular}

\section{OR (multivariate)}

$0.70(0.43-0.92, \mathrm{p}=0.047)$

$0.92(0.80-1.04, \mathrm{p}=0.201)$

$1.07(0.81-1.44, \mathrm{p}=0.642)$

$1.18(0.85-1.62, \mathrm{p}=0.249)$

$1.06(0.84-1.53, \mathrm{p}=0.672)$

$1.04(0.90-1.21, \mathrm{p}=0.572)$

$0.54(0.04-6.23, \mathrm{p}=0.611)$

In a multivariate model that included other right functional parameters such as TAPSE, S-wave, estimated pulmonary artery systolic pressure and LVEF, RV free wall strain maintained its relation to cardiovascular mortality at 6 months.

\begin{tabular}{|c|l|rrrr|}
\hline CVdeath6m & & no & \multicolumn{1}{c}{ yes } & OR (univariate) & OR (multivariate) \\
\hline RVFWLS & Mean (SD) & $17.7(4.3)$ & $14.7(4.6)$ & $0.86(0.73-1.00, \mathrm{p}=0.049)$ & $0.80(0.63-0.98, \mathrm{p}=0.043)$ \\
\hline PASP & Mean (SD) & $46.6(14.9)$ & $57.2(22.7)$ & $1.03(0.99-1.08, \mathrm{p}=0.088)$ & $1.04(0.99-1.10, \mathrm{p}=0.168)$ \\
\hline TAPSE & Mean (SD) & $19.0(3.4)$ & $19.0(3.4)$ & $1.00(0.80-1.21, \mathrm{p}=0.972)$ & $1.26(0.82-2.07, \mathrm{p}=0.309)$ \\
\hline S & Mean (SD) & $11.1(2.4)$ & $10.3(1.3)$ & $0.84(0.56-1.16, \mathrm{p}=0.337)$ & $0.44(0.16-0.96, \mathrm{p}=0.072)$ \\
LVEF & Mean (SD) & $54.3(8.7)$ & $59.5(7.0)$ & $1.08(0.99-1.18, \mathrm{p}=0.090)$ & $1.14(1.01-1.35, \mathrm{p}=0.052)$ \\
\hline
\end{tabular}

LVGLS and E/e' ratio were related to cardiovascular mortality at 6 months regardless of the ejection fraction or other clinical parameters related to mortality such as BMI, age, FA, kidney failure, COPD and ischemic heart disease (IC).

\begin{tabular}{|c|l|r|r|}
\hline CVdeath6m & & \multicolumn{1}{c|}{ no } & \multicolumn{1}{c|}{ yes } \\
\hline Age & Mean (SD) & $77.0(10.9)$ & $81.2(9.3)$ \\
\hline LVGLS & Mean (SD) & $14.7(3.3)$ & $11.9(2.9)$ \\
\hline LVEF & Mean (SD) & $54.3(8.7)$ & $59.5(7.0)$ \\
\hline BMI & Mean (SD) & $29.5(4.8)$ & $32.5(6.7)$ \\
\hline AF & no & $28(87.5)$ & $4(12.5)$ \\
\hline & yes & $33(84.6)$ & $6(15.4)$ \\
\hline KF & no & $41(93.2)$ & $3(6.8)$ \\
\hline & yes & $20(74.1)$ & $7(25.9)$ \\
\hline IC & no & $49(84.5)$ & $9(15.5)$ \\
& yes & $12(92.3)$ & $1(7.7)$ \\
\hline COPD & no & $50(87.7)$ & $7(12.3)$ \\
& yes & $11(78.6)$ & $3(21.4)$ \\
\hline E/e' & Mean (SD) & $17.1(7.3)$ & $25.1(9.1)$ \\
\hline
\end{tabular}

OR (univariate)
$1.04(0.98-1.13, \mathrm{p}=0.250)$
$0.76(0.59-0.95, \mathrm{p}=0.021)$
$1.08(0.99-1.18, \mathrm{p}=0.090)$
$1.11(0.98-1.26, \mathrm{p}=0.101)$
-
$1.27(0.33-5.40, \mathrm{p}=0.729)$
-
$4.78(1.19-24.06, \mathrm{p}=0.035)$
-
$0.45(0.02-2.78, \mathrm{p}=0.473)$
-
$1.95(0.38-8.31, \mathrm{p}=0.384)$
$1.11(1.03-1.22, \mathrm{p}=0.015)$

\section{OR (multivariate)}

0.95 (0.84-1.07, $\mathrm{p}=0.377$ )

$0.60(0.34-0.91, \mathrm{p}=0.034)$

$1.16(1.01-1.39, \mathrm{p}=0.076)$

$0.99(0.78-1.22, \mathrm{p}=0.933)$

$0.93(0.09-9.97, \mathrm{p}=0.949)$

$1.36(0.16-11.38, \mathrm{p}=0.767)$

$1.68(0.06-30.94, \mathrm{p}=0.726)$

$2.67(0.21-31.36, \mathrm{p}=0.424)$

$1.14(1.01-1.32, \mathrm{p}=0.045)$ 


\section{DISCUSSION}

In several studies, it has been observed that patients with heart failure with preserved ejection fraction, an altered right ventricular function is common and represents a relevant prognostic factor $(1,9,11)$. Right ventricular function can be affected in patients with heart failure by the same processes that cause left ventricular dysfunction (12).

The special morphology and arrangement of the right ventricle make the evaluation by echocardiogram a challenging task. The RV chamber is difficult to evaluate by two-dimensional echocardiography because of its complex structure. Small variations in the plane acquisition modify the measurements significantly and generate changes in the measurement of the RV function indices. Generally, measurements of the RV are less reproducible and accurate than those of the $\mathrm{LV}$, and there are more available methods of functional quantification for the LV than the RV. Several methods have been proposed to assess RV function with echocardiography: fractional area change (FAC), tricuspid annular plane systolic excursion (TAPSE), lateral tricuspid annular systolic velocity derived from tissue Doppler (S') and Tei index (5). These indices are very useful but have the important limitation of depending on the insonation angle thus their value can be affected by translational and rotational motions of the heart, especially present in the right ventricle (6).

The use of techniques for studying RV deformation by echocardiography (global longitudinal strain) can provide useful information in the assessment of RV function quickly and non-invasively. The assessment of RV function using right ventricular free wall longitudinal deformation is carried out using speckle tracking programs of the three segments of the RV free wall. The lower limit of normality has been set to $<-20 \%$ (greater than $20 \%$ in absolute value) $(7,8)$. The technique was developed in a similar way to that used for the study of left ventricular function $(13,14)$.

The usefulness of right ventricular deformation parameters by determining the RV free wall speckletracked strain has been evaluated and compared with other right functional parameters. Thus, in a study of Fukuda and collaborators, in 49 patients with pulmonary hypertension, RV free wall strain was observed to be significantly lower than that of healthy control subjects, and there was a correlation with right ventricular functional parameters such as the RV ejection fraction calculated by magnetic resonance imaging, and the functional status of the patient (15). It has been observed that the degree or duration of right ventricular pressure overload does not fully explain right ventricular dysfunction in HFpEF (16).

In the same way that different comorbidities such as HBP (high blood pressure), diabetes mellitus, obesity and coronary artery disease directly affect longitudinal systolic and diastolic function of the left ventricle as a consequence of fibrotic subendocardial change $(17,18)$, they can also affect the subendocardial fibers of the right ventricle (19). Authors such as Kosmala and collaborators observed right ventricular dysfunction in diabetic patients with preserved ejection fraction, with no difference in TAPSE or S wave (20). Diabetes is one of the main causes of HfpEF, mainly because of a greater presence of ischemic heart disease, although multiple pathophysiological mechanisms have also been implicated. A metabolic toxic effect on cardiomyocytes has been postulated among other reasons. In our study, we observed a high prevalence of DM (46\%) in our patients, as they correspond to the group of patients with preserved and mid-range EF. Right ventricular longitudinal dysfunction has also been evidenced in patients with hypertension (21), supporting the theory of involvement of both ventricles (not only the LV, but also the $\mathrm{RV}$ ) in relation to HBP and other comorbidities such as DM that would cause a global effect on myocardial cells.

There is most likely to be an association with the same fibrosis process that affects the subendocardial layer of the $\mathrm{LV}$ and also to a lesser extent with the pressure overload secondary to pulmonary 
hypertension. The arrangement of the myocardial fibers in the right ventricle is predominantly longitudinal. There is a predominant longitudinal subendocardial layer and a thinner circumferential subepicardial layer. Thus, the process of right ventricular contraction and relaxation occur fundamentally in the longitudinal direction, this component being the one that contributes the most to the right ventricular ejection volume, much more than that of the contraction movement of the circumferential fibers (22).

In our study, a significant correlation between LVGLS and free wall RVLS was observed, which suggests biventricular involvement of the myocardium, therefore ventricular longitudinal functional affection would occur in both left and right ventricles simultaneously.

Our results through longitudinal strain revealed right ventricular dysfunction in HFpEF and HFmrEF with a worse clinical outcome. However, other functional parameters such as TAPSE and FAC were not as altered nor were they related to higher HF readmission or cardiovascular mortality. Maintaining normal TAPSE in HFpEF patients is not synonymous with normal RV function. TAPSE is a regional indicator that describes longitudinal function of the RV at lateral basal level and can be affected by load changes such as volumetric overload. Despite its wide utilization and proven prognostic value, it could be a less sensitive indicator of ventricular dysfunction than free wall strain. Fractional area change calculation is dependent of a good image quality and correct acquisition of the RV centric plane with meticulous endocardial border delineation (that is more trabeculated than the LV). It is also time consuming and cannot always be obtained reliably.

There is less data available on the Tei Index in RV functional assessment in this patient population. Tei index has been widely used for the study of left ventricular dysfunction, as it is an easy to apply tool for ventricular dysfunction (25). Its main disadvantage is the angle dependence and that it does not differentiate systolic from diastolic dysfunction. As it requires several measurements for its calculation, it can constitute a potential source of error if one or more erroneous measurements are chained together, a weakness common in a large number of echocardiographic formulas.

When image quality is adequate and endocardial border detection is correct, RV free wall longitudinal strain is quantified semi-automatically with less chance of error than other parameters that require more measurements. It is a quick application tool, rather than the LVGLS, which requires three planes for its determination. Although it is also necessary to pay attention to technical aspects, such as the adjustment of the width of the region of interest, since the RV wall is thinner than that of the LV.

\section{LIMITATIONS}

Our study has several limitations. One is that the RV function was evaluated by transthoracic echocardiography. Other more accurate and objective methods such as magnetic resonance imaging (gold standard) would have increased the reliability of the study in assessing right ventricular function. The availability of echocardiography is greater in clinical practice and its use much more widespread. Progressive improvement of the technology applied to echocardiography allows the assessment of increasingly accurate and reproducible measurements, even more so with the incorporation of semiautomatic methods as in the case of strain.

Another study limitation is sample size (71 patients). Not all patients are eligible due to suboptimal windows for reliable strain determination. To a large extent the presence of characteristic comorbidities in HF with mid-range and preserved LVEF such as obesity and COPD, limit the image quality through transthoracic access. The characteristics of patients in our sample (advanced age) may not be applicable to the younger population, however heart failure with preserved ejection fraction affects increasingly 
elderly patients in western countries and no clinically relevant differences in strain have been established in the literature depending on age (26).

The determination of RV strain requires taking care of technical aspects for its correct calculation. In patients with atrial fibrillation and therefore with an irregular RR interval, the measurement of strain has the limitation of representing cycles of different lengths. In these cases, an average of three beats is used. In our study, the calculation of right ventricular strain corresponds to the RV free wall strain, excluding septal segments. It has been observed that the interventricular septum also contributes to right ventricular performance although to a lesser extent than the free wall, representing up to $20-40 \%$ of the increase in right ventricular pressure in healthy subjects (27).

However, most studies carried out under various pathological conditions refer to the right ventricular free wall strain $(26,28)$ and in our target population (heart failure) left ventricular dysfunction may condition a reduction in longitudinal strain of septal segments affecting the determination of six-segment RV strain secondary to left failure.

\section{CONCLUSIONS:}

In patients with heart failure with preserved and mid-range ejection fraction, impairment of right ventricular free wall strain is common and is related to worse clinical outcome (increased cardiovascular mortality at six months) regardless of other right ventricular functional parameters and left ventricular ejection fraction. Therefore, representing a sensitive non-invasive prognostic indicator in these patients, and could be useful in stratifying the risk of adverse events. RV and LV strain are correlated indicating biventricular involvement of deformation parameters with prognostic significance.

\section{BIBLIOGRAPHY}

1 Melenovsky V, Hwang SJ, Lin G, Redfield MM, Borlaug BA. Right heart dysfunction in heart failure with preserved ejection fraction. Eur Heart J 2014;35:3452-3462.

2. Mohammed SF, Hussain I, AbouEzzeddine OF, Abou Ezzeddine OF, Takahama

H, Kwon SH, Forfia P, Roger VL, Redfield MM. Right ventricular function in heart failure with preserved ejection fraction: a community-based study. Circulation 2014;130:2310-2320.

3 Moreira $\mathrm{H}$ et al .Right ventricular systolic dysfunction in Chagas disease defined by speckletracking echcardiography: a comparative study with cardiac magnetic resonance imaging. JASE. 2017;30 (5): 493-502.

4 Kaul S, TeiC, Hopkins JM, ShahPM. Assessment of right ventricular function using twodimensional echocardiography. Am Heart J 1984;107:526-31.

5 Rudski LG, Lai WW, Afilalo J, Hua L, Handschumacher MD, Chandrasekaran K et al. Guidelines for the echocardiographic assessment of the right heart in adults: A report from the American Society of Echocardiography endorsed by the European Association of Echocardiography, a registered branch of the European Society of Cardiology, and the Canadian Society of Echocardiography. J Am Soc Echocardiogr 2010; 23: 685-713. 
6 Barón A. Deformación de la pared libre del ventrículo derecho: evaluación no volumétrica de la función. Rev Colomb Cardiol. 2012;19 (5):223-224

7 Lang RM, Badano LP, Mor-Avi V, Afilalo J, Armstrong A, Ernande L, et al.

Recommendations for cardiac chamber quantification by echocardiography in adults. J Am Soc Echocardiogr. 2015 Jan;28(1):1-39.

8 Teske AJ, De Boeck BW, Olimulder M, Prakken NH, Doevendans AF, Cramer MJ. Echocardiographic assessment of regional right ventricular function: a head-to-head comparison between 2-dimensional and tissue Doppler derived strain analysis. J Am Soc Echocardiogr 2008;21:275-83

9 Morris DA, Gailani M, Vaz Pérez A, et al. Right ventricular myocardial systolic and diastolic dysfunction in heart failure with normal left ventricular ejection fraction. J Am Soc

Echocardiogr. 2011;24(8):886-897

10 Damman K, Navis G, Smilde TD, Voors AA, van der Bij W, van Veldhuisen DJ, et al. Decreased cardiac output, venous congestion and the association with renal impairment in patients with cardiac dysfunction. Eur J Heart Fail 2007;9:872-8.

11 Obokata M, Reddy YNV, Melenovsky V, Pislaru S, Borlaug BA. Deterioration in right ventricular structure and function over time in patients with heart failure and preserved ejection fraction. Eur Heart J. 2019;40(8):689-697.

12 Cikes, M, Solomon S.An integrative approach for assessment of cardiac function in HF. Eur Heart J. 2016. Jun 1;37(21):1642-50

13 Meris A, Faletra F, Conca C, Klersy C, Regoli F, Klimusina J, et al. Timing and magnitude of regional right ventricular function: a speckle trackingderived strain study of normal subjects and patients with right ventricular dysfunction. J Am Soc Echocardiogr 2010;23:823-31

14 D’Andrea A, Caso P, Bossone E, Scarafile R, Riegler L, Di Salvo G, et al. Right ventricular myocardial involvement in either physiological or pathological left ventricular hypertrophy: an ultrasound speckle-tracking twodimensional strain analysis. Eur J Echocardiogr 2010;11:492500 .

15 Fukuda Y, Tanaka H, Sugiyama D, et al. Utility of right ventricular free wall speckletracking strain for evaluation of right ventricular performance in patients with pulmonary hypertension. J Am Soc Echocardiogr. 2011;24(10):1101-1108.

16 Bogaard HJ, Natarajan R, Henderson SC, Long CS, Kraskauskas D,Smithson L, et al. Chronic pulmonary artery pressure elevation is insufficient to explain right heart failure. Circulation 2009;120:1951-60

17 Ng AC, Delgado V, Bertini M, van der Meer RW, Rijzewijk LJ, Shanks M, et al. Findings from left ventricular strain and strain rate imaging in asymptomatic patients with type 2 diabetes mellitus. Am J Cardiol 2009;104: 1398-401. 
18 Stanton T, Marwick TH. Assessment of subendocardial structure and function. J Am Coll Cardiol Img 2010;3:867-75

19 Broberg CS, Chugh SS, Conklin C, Sahn DJ, Jerosch-Herold M. Quantification of diffuse myocardial fibrosis and its association with myocardial dysfunction in congenital heart disease. Circ Cardiovasc Imaging 2010; 3:727-34.

20 Kosmala W, Colonna P, Przewlocka-Kosmala M, Mazurek W. Right ventricular dysfunction in asymptomatic diabetic patients. Diabetes Care. 2004;27(11):2736-2738

21. Cicala S, Galderisi M, Caso P, et al. Right ventricular diastolic dysfunction in arterial systemic hypertension: analysis by pulsed tissue Doppler. Eur J Echocardiogr 2002;3:135-42

22 Leather HA, Ama' R, Missant C, Rex S, Rademakers FE, Wouters PF. Longitudinal but not circumferential deformation reflects global contractile function in the right ventricle with open pericardium. Am J Physiol Heart Circ Physiol 2006;290:H2369-75

23 Damman K, Navis G, Smilde TD, Voors AA, van der Bij W, van Veldhuisen DJ, et al. Decreased cardiac output, venous congestion and the association with renal impairment in patients with cardiac dysfunction. Eur J Heart Fail 2007;9:872-8.

24 Testani JM, Khera AV, St John Sutton MG, Keane MG, Wiegers SE, Shannon RP, et al. Effect of right ventricular function and venous congestion on cardiorenal interactions during the treatment of decompensated heart failure. Am J Cardiol 2010;105:511-6.

25 Goroshi M, Chand D. Myocardial Performance Index (Tei Index): A simple tool to identify cardiac dysfunction in patients with diabetes mellitus. Indian Heart J. 2016;68(1):83-87

26 Muraru D, Onciul S, Peluso D, et al. Sex- and Method-Specific Reference Values for Right Ventricular Strain by 2-Dimensional Speckle-Tracking Echocardiography. Circ Cardiovasc Imaging. 2016;9(2):e003866

27 Buckberg GD; RESTORE Group. The ventricular septum: the lion of right ventricular function, and its impact on right ventricular restoration. Eur J Cardiothorac Surg. 2006;29(suppl 1):S272-S278.

28 Antoni ML, Scherptong RW, Atary JZ, Boersma E, Holman ER, van der Wall EE, Schalij MJ, Bax JJ. Prognostic value of right ventricular function in patients after acute myocardial infarction treated with primary percutaneous coronary intervention. Circ Cardiovasc Imaging. 2010;3:264-271 



\section{Figures}

\section{8/06/2019 18:37:24 \\ $18: 37: 24$}

VI A4C 1/1

18:37:26

rC = 16 Ipm

DC tmpo, reg. .... ms

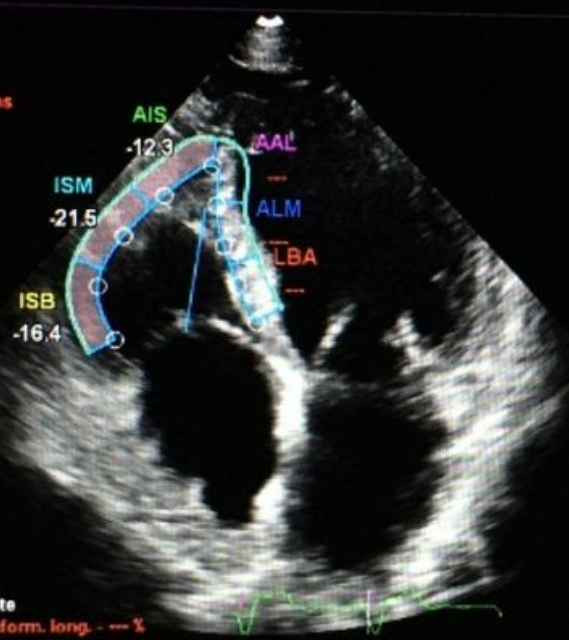

Aceptación pendiente VI AAC, Tndo Pico Deform. long - - - 8
$20 x$

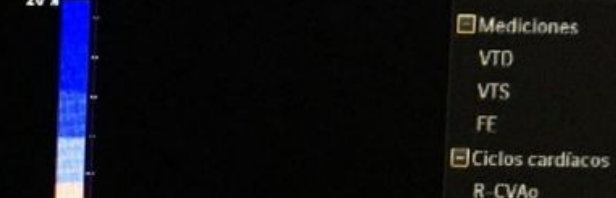

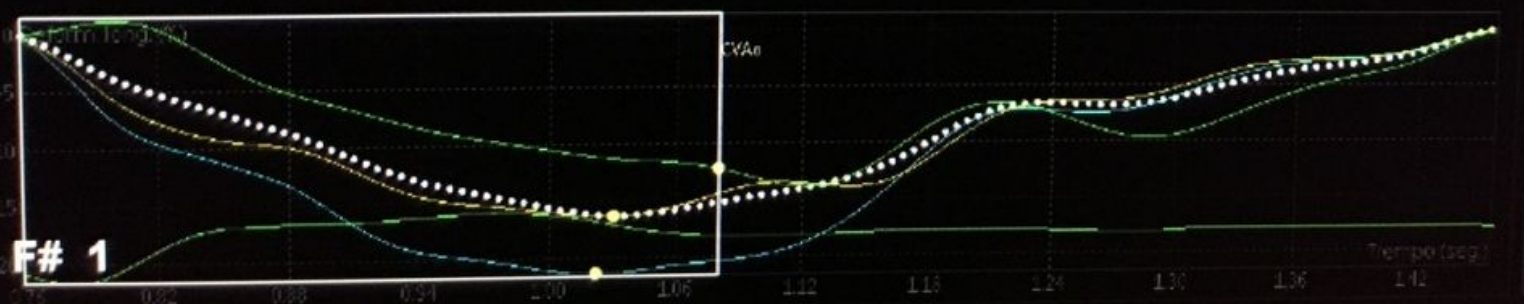

\section{Figure 1}

Determination of the right ventricular free wall strain through two-dimensional speckle tracking echocardiogram 


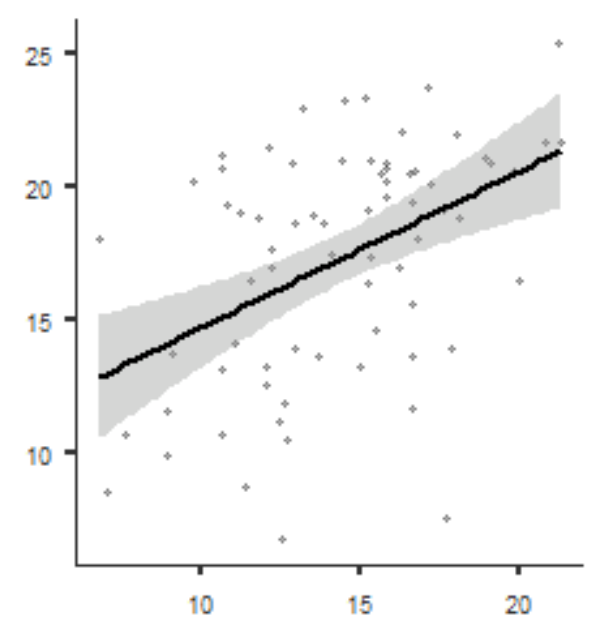

\section{Figure 2}

In the linear regression analysis, significant $(p<0.01)$ linear correlation (Pearson $r 0.45, \mathrm{Cl} 0.246-0.619)$ was observed between the longitudinal deformation indices of both ventricles (Figure 2) 